\title{
Conocimientos, practicas y habilidades sobre la búsqueda bibliográfica y percepción estudiantil sobre la capacitación universitaria en investigación, en estudiantes de obstetricia
}

\author{
Mauro Huamani-Navarro, Diana Alegría-Delgado, Milena López-Sánchez, Carolina B. Tarqui-Mamani, \\ Luis Ormeño-Caisafana
}

Introducción. Se observan estudiantes del pregrado de obstetricia con insuficientes habilidades para utilizar toda la información que necesitan. Por ello, nos proponemos determinar el nivel de conocimiento, las prácticas y habilidades sobre la búsqueda de información en Internet, además de la percepción estudiantil sobre la capacitación brindada por la universidad y sobre su nivel en investigación.

Sujetos y métodos. Estudio descriptivo transversal, periodo abril-julio de 2009, en 150 estudiantes de obstetricia de la Facultad de Medicina de la Universidad Nacional Mayor de San Marcos, en Perú. Por medio de un cuestionario estructurado, se realizó un análisis univariado.

Resultados. El 78,7\% presentó conocimientos bajos; el 55,3\%, prácticas incorrectas, y el $74,7 \%$, deficientes habilidades para realizar una búsqueda. El 60\% consideraba que la preparación en búsqueda de información era regular o deficiente, con igual proporción para los procesos de publicación, redacción, lectura crítica y metodología de la investigación.

Conclusiones. Identificar los conocimientos, prácticas, habilidades y percepciones sobre la investigación es importante para promover programas de capacitación extracurricular en los estudiantes durante el pregrado.

Palabras clave. Acceso a la información. Bases de datos bibliográficas. Estudiantes de obstetricia. Internet. Investigación.

Facultad de Medicina; Universidad Nacional Mayor de San Marcos (M. López-Sánchez, C.B. TarquiMamani, L. Ormeño-Caisafana). Sociedad Científica de Estudiantes y de Obstetricia de San Marcos (M. Huamani-Navarro, D. AlegríaDelgado, L. Ormeño-Caisafana). Lima, Perú.

Correspondencia: Obs. Mauro Huamani Navarro. Jr. Honduras, 398 Comas, Lima 32, Perú.

E-mail:

hnmauro@gmail.com

(c) 2011 Educación Médica

Know-how, practicals and skills concerning bibliographical searches and students' perception of university training in research, in the case of students of obstetrics

Introduction. Undergraduate students of midwifery were observed with insufficient skills to use all the information they need. Therefore, we propose to determine the level of knowledge, practices and skills in finding information on the Internet, as well as student perceptions about the training provided by the university and research level.

Subjects and methods. Descriptive study period April-July 2009, 150 students of midwifery of the Faculty of Medicine of the Universidad Nacional Mayor de San Marcos in Peru. Through a structured questionnaire, we conducted a univariate analysis.

Results. $78.7 \%$ had low knowledge, 55.3\%, malpractice, and $74.7 \%$, impaired ability to perform a search. $60 \%$ felt that the preparation in search of information was fair or poor, with equal proportion to the publishing process, writing, critical reading and research methodology.

Conclusions. Identify the knowledge, practices, skills and perceptions of the research is important to promote extracurricular training programs for undergraduate students.

Key words. Access to the information. Bibliographical databases. Internet. Research. Students of midwifery.

\section{Introducción}

En la formación biomédica adquiere cada vez más importancia la alfabetización informacional [1,2]. Tener alfabetización informacional es saber cuándo y por qué necesitar información, dónde se ubica y cómo recuperarla, evaluarla, utilizarla y comunicarla. Se relaciona con el enfoque constructivista del aprendizaje y contribuye a que la persona logre un aprendizaje significativo [2]. La alfabetización informacional no sólo es instrucción en habilidades de búsqueda, sino desarrollar competencias para usar 
la información en la toma de decisiones y generar conocimiento; implica desarrollar habilidades cognitivas y éticas. La alfabetización informacional es parte necesaria de la gestión del conocimiento, cuyo objetivo en salud consiste en mejorar la prestación, lograr la equidad, la eficiencia y aumentar el nivel de salud de la población [3]. En la actualidad, con las tecnologías de la información y la comunicación (TIC), revistas electrónicas, bibliotecas virtuales, historias clínicas electrónicas, etc., se podría afirmar que en salud se hace gestión del conocimiento de forma eficaz, al menos en sus aspectos básicos, pero no es así. La información científica biomédica actual se ubica en su mayoría en bases de datos virtuales como Pubmed o Scielo, entre muchas otras, con progresivos incrementos en sus publicaciones [4-9]. Las necesidades de información deberían estar cubiertas, pero numerosa información no resulta útil para la práctica clínica ya que es de desigual calidad o redundante [3]. Las fuentes no especializadas (Google y otras) complican el problema [10] y la situación empeora con la falta de filtros editoriales y sistemas de evaluación de las páginas web, lo que afecta a profesionales y pacientes, quienes se exponen a información de calidad dudosa $[11,12]$. Se estima que las páginas web se incrementarán aún más $[10,13]$ y esto preocupa por el riesgo que implica la propagación de información sin control de calidad [11]. Por otro lado, pese a que en los estudiantes y profesionales de la salud se ha extendido el uso de Internet [14] y se tienen percepciones positivas acerca de su aplicación para la práctica clínica, muchos presentan insuficientes habilidades para acceder, recuperar, ubicar y utilizar toda la información que necesitan $[14,15]$. Este problema no se reduce a la simple búsqueda, sino al adecuado manejo de dicha información, es decir, a su lectura, interpretación y aplicación [16-18]. Diversos estudios en Latinoamérica muestran esta realidad, la carencia de habilidades básicas para la búsqueda y uso de la información científica en profesionales de salud [19, 20], es decir, deficiente alfabetización informacional. Contribuyen a este problema los usos más frecuentes que los estudiantes hacen de Internet no relacionados con la investigación (chats, redes sociales, etc.) $[21,22]$, la exigua promoción de dichas habilidades durante la formación en el pregrado a través de programas formales y extracurriculares y, por consiguiente, la percepción que los estudiantes tienen sobre la capacitación que brinda la universidad en investigación [23]. Estos aspectos pasan a ser causa multifactorial de la baja producción científica estudiantil [24,25]. Por ello, en este estudio, nos proponemos determinar el nivel de conocimiento, prác- ticas y habilidades sobre la búsqueda de información en Internet, e identificar la percepción estudiantil sobre la capacitación brindada en investigación por la universidad y la percepción sobre su nivel de competencia en el proceso de investigación.

\section{Sujetos y métodos}

Se realizó un estudio transversal y descriptivo, en el que se incluyó a 383 estudiantes matriculados en la Escuela de Obstetricia de la Facultad de Medicina de la Universidad Nacional Mayor de San Marcos, durante el periodo abril-julio del año 2009. La muestra calculada fue de 165 estudiantes seleccionados de las listas de matriculación; el muestreo empleado fue probabilístico y estratificado, para lo cual se consideraron los siguientes criterios: intervalo de confianza del 95\%, precisión del 5\% y $p=20 \%(k s h=$ 0,3838; $k=3$ ). Del total de estudiantes, sólo respondieron 150 estudiantes (tasa de rechazo: 15\%).

A cada estudiante se le aplicó un cuestionario estructurado, autodesarrollado, anónimo, previo consentimiento informado verbal. El cuestionario presentó tres secciones:

- Datos generales (edad, sexo, semestre de estudio, año de ingreso, curso de investigación llevado, pertenencia a una sociedad científica y realización de un trabajo de investigación).

- Conocimientos, percepciones, prácticas y habilidades sobre búsqueda de información en internet.

- Apreciación sobre la capacitación recibida y su nivel actual en el proceso de investigación, evaluada mediante una escala tipo Likert.

El cuestionario fue desarrollado por los autores y para ello previamente se recabaron diversos cuestionarios con objetivos similares. Se realizó un estudio piloto para evaluar la estructura interna del cuestionario en 20 estudiantes de primer a cuarto año y se modificó el cuestionario de acuerdo con los resultados. La calificación de las variables se realizó por criterio de los autores, pero en base a la búsqueda bibliográfica [15,21-23]. La calificación de las percepciones se realizó en base a tres preguntas que buscaban identificar cómo los estudiantes perciben sus conocimientos (percepciones buenas, regulares o malas). En la calificación de los niveles de conocimientos se siguió un procedimiento similar en base a las respuestas de tres preguntas, pudiendo ser su nivel de conocimiento alto (tres respuestas correctas), intermedio (dos respuestas correctas) o bajo (una respuesta correcta). De igual forma se procedió con las prácticas, calificándose como práctica correcta 
aquella en la cual el estudiante busca información por iniciativa propia, utiliza bases de datos especializadas y visita al menos tres bases de datos en el último mes, de una lista de bases (Scielo, Lilacs, Biblioteca Virtual de Salud, Pubmed/Medline, Cochrane, Hinari). La búsqueda por indicación del profesor, el uso de Google, el no uso de bases de datos y el no visitar al menos tres bases de datos en el último mes calificaban las prácticas como incorrectas. Finalmente, se calificaron las habilidades de los estudiantes para redactar la forma como realizaban una búsqueda, calificándose como aquel que 'sabe hacer una búsqueda' si escribe el sitio o el nombre de la base de datos, determina la estrategia de búsqueda y menciona cómo hacerla. De lo contrario, si estableció pasos mediante los cuales no podría realizar una búsqueda o sólo señaló vías no adecuadas, fue calificado como 'no sabe hacer una búsqueda'. En cuanto a la apreciación sobre la capacitación que recibió durante su formación en pregrado y el nivel actual que posee en materia de investigación, se incluían los siguientes ítems: proceso de publicación, lectura crítica, redacción de artículos, búsqueda de información y metodología de la investigación, que se valoraron mediante una escala tipo Likert (nula, deficiente, regular, buena, muy buena). Los datos recogidos se tabularon y analizaron mediante el programa SPSS v. 15.0, con un nivel de significación del $5 \%$ y un intervalo de confianza del 95\% (IC 95\%). Se realizó el análisis descriptivo, que incluyó cálculo de promedios, desviación estándar y distribución de frecuencias de las variables, en porcentajes y frecuencias.

\section{Resultados}

El promedio de edad de los estudiantes fue de 21,1 $\pm 2,1$ años (IC 95\% = 20,7-21,4 años) y fluctúo entre 27 y 16 años, con predominio de estudiantes de sexo femenino ( $88 \% ; n=132$ ). La proporción por semestre de estudio fue del $19 \%(n=29)$ para el internado y del $20 \%(n=31)$ para los estudiantes del primer al cuarto año. El 59,7\% $(n=89)$ refirió haber recibido el curso de metodología de la investigación, y el 3,3\% $(n=8)$, pertenecer a alguna sociedad científica estudiantil. Respecto a la producción científica, el 66,9\% $(n=88)$ refirió haber hecho alguna investigación.

\section{Conocimientos sobre la búsqueda de información en Internet}

Del total de estudiantes encuestados, el 18,7\% $(n=28)$ conocía correctamente los pasos a realizar para una
Figura 1. Bases de datos visitadas en los últimos 30 días por estudiantes de ciencias de la salud.

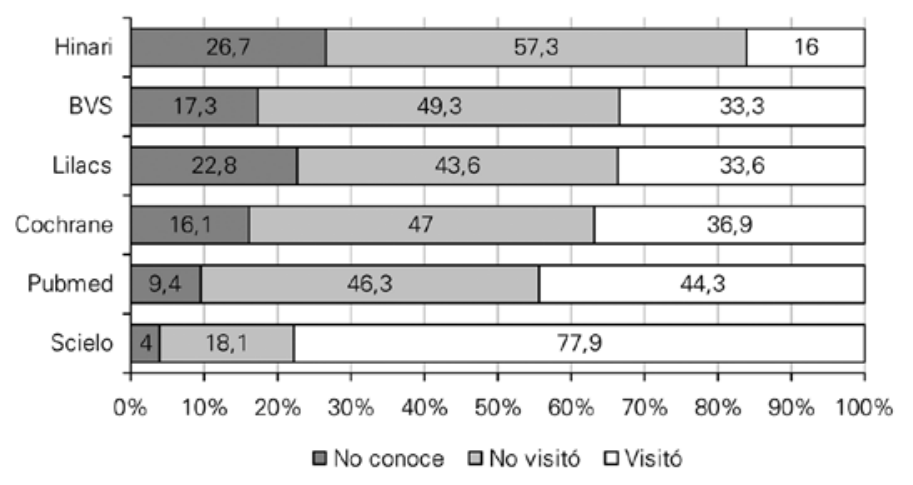

búsqueda simple; el 18,7\% $(n=28)$ conocía correctamente los operadores boleanos, y el 35,3\% $(n=53)$ sabía correctamente las estrategias de búsqueda bibliográfica. En el ítem que evaluó los conocimientos acerca de las estrategias de búsqueda, el 35,3\% $(n=53)$ respondió de forma correcta. La evaluación global mostró que el 78,7\% $(n=118)$ de estudiantes poseía un conocimiento bajo; el 18,0\%, conocimientos medios, y sólo el 3,0\%, conocimientos altos. Adicionalmente, el 78,0\% $(n=117)$ afirmó conocer los pasos para hacer una búsqueda, pero había trabajado poco con ellos. El 48,6\% $(n=72)$ percibía conocer medianamente las estrategias para la búsqueda de información, y el 69,3\%, conocer medianamente los principales sitios de Internet en donde es posible obtener información fiable. La evaluación global mostró que el 20,7\% presentaba percepciones buenas con respecto a sus conocimientos, y el resto, una percepción baja o regular.

\section{Prácticas}

El 63,0\% $(n=92)$ de estudiantes buscaban información en salud por iniciativa propia, mientras que el $37,0 \%(n=54)$, por indicación del profesor. El 42,0\% $(n=63)$ de estudiantes prefería realizar la búsqueda a través de Google, el 44,0\% $(n=66)$ utilizaba alguna base de datos para su búsqueda, mientras que el $14,0 \%(n=21)$ no usaba una base de datos ni el buscador Google como fuente de información. El 54,7\% de estudiantes había visitado menos de tres de las bases de datos presentadas. De acuerdo con la evaluación global de las prácticas, el 55,3\% de los estudiantes poseía prácticas incorrectas sobre la búsqueda de información en Internet (Fig. 1). 
Figura 2. Percepciones sobre la capacitación recibida durante su formación.

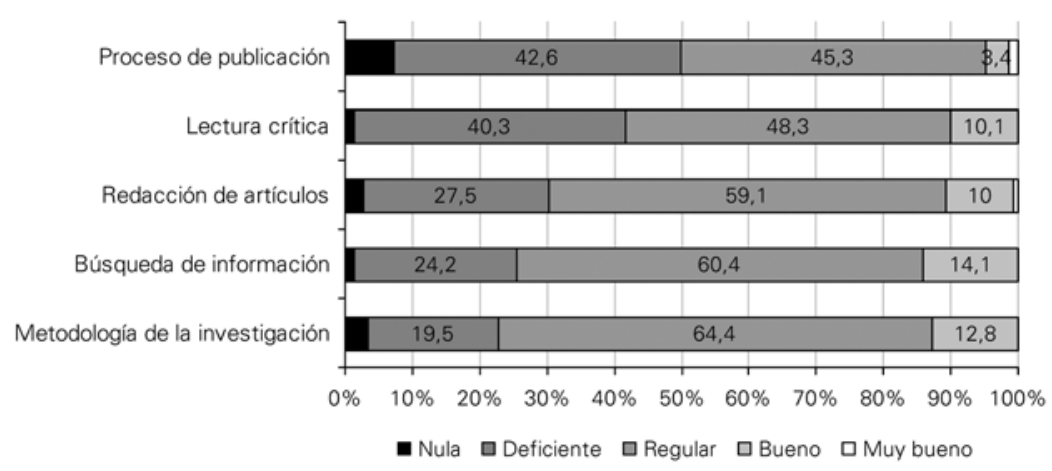

Figura 3. Nivel actual en el proceso de la investigación.

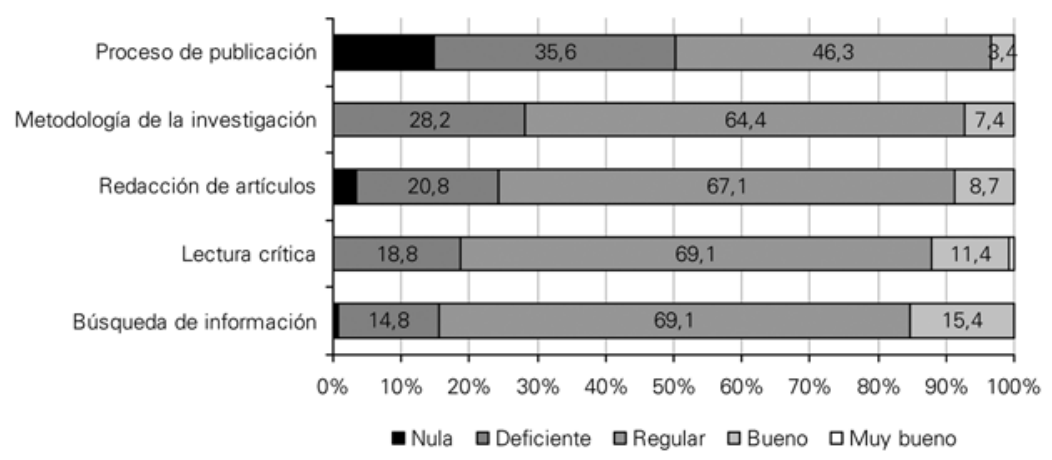

Con respecto a la habilidad, se evalúo la forma de buscar información, y para ello se tomó en cuenta el uso de descriptores para la búsqueda de información. Se observó que el 74,7\% 'no sabía hacer una búsqueda', el 20,0\% 'sabía hacer medianamente' la búsqueda, y sólo el 5,3\% 'sabía hacer una búsqueda de forma correcta?

\section{Percepción estudiantil sobre la capacitación brindada en investigación por la universidad}

El 12,8\% de los estudiantes percibía haber recibido una buena capacitación en metodología de la investigación. El 14,1\% percibía como buena la capacitación recibida en temas sobre búsqueda de información; el 3,4\% percibía haber recibido una buena capacitación en el proceso de publicación, y el 10,1\%, en lectura crítica y redacción de artículos (Fig. 2).
Percepción estudiantil sobre su nivel de competencia en el proceso de investigación

El 7,4\% de estudiantes consideraba bueno su nivel actual en metodología de la investigación; el 15,4\% valoraba como bueno su nivel en búsqueda de información, y en cambio sólo el 3,4\%, 11,4\% y 8,7\% consideraba bueno su nivel actual en el proceso de publicación, lectura crítica y redacción de artículos, respectivamente (Fig. 3).

\section{Discusión}

La revisión de la bibliografía es un componente importante del proceso de investigación y las habilidades para realizar búsquedas especializadas son esenciales para cada momento del mismo. Pese a la diferente metodología empleada para estudiar este problema, nuestros hallazgos -estudiantes con bajos conocimientos $(78,7 \%)$, con prácticas incorrectas $(55,3 \%)$ y con deficientes habilidades $(74,7 \%)$ para realizar una búsqueda en Internet- reflejan la magnitud y vigencia del problema, al igual que lo descrito por González y Santana-Arroyo [21] y por Horna et al [22] respecto a deficiencias en las habilidades y escasa cultura científica en el uso de Internet. La situación que atraviesan los estudiantes, al parecer, es parte de una continua carencia de habilidades que se expresará durante la practica clínica y la actividad científica de los profesionales de salud $[15,19,20]$. Esto exige continuar su estudio e identificar los factores asociados a la alfabetización informacional.

A pesar de que la universidad realiza esfuerzos por adquirir licencias y convenios que permitan el acceso a bases de datos científicas, se observa que los estudiantes prefiere buscar información haciendo uso de Google $(42,0 \%)$ y no utilizan bases de datos especializadas como Scielo (22,1\%), Lilacs (66,4\%), Pubmed (55,7\%), Cochrane (63,1\%) y Hinari (84\%), o alguna página de tránsito como la Biblioteca Virtual de Salud (66,6\%), pese a que estas bases son de acceso libre y de reconocido prestigio por la comunidad científica, lo que muestra una subutilización de estos recursos. Además, se observó que las fuentes más consultadas corresponden a publicaciones de la región (Scielo, Lilacs, Redalyc, etc.), que representan sólo un $2 \%$ de la producción científica mundial. Existen fuentes de información que no se han considerado en el estudio (Embase Experta, Ovid...) y que junto a las herramientas de la web 2.0 (redes sociales, wikis, blogs, etc.), los sistemas de alertas, las revistas secundarias, etc., son importantes a considerar en estudios futuros [5], pero con- 
llevan la siguiente pregunta: si aún no maximizamos el uso de la tecnología de Internet disponible y ya estamos pasando a otro contexto informático, ¿cómo se garantizará la preparación integral que se busca en todo profesional de la salud? Está clara la necesidad de desarrollar habilidades en alfabetización informacional, pues en caso contrario se corre el riesgo de asfixiarse ante la cantidad de información biomédica que aumenta constantemente y que evidencia que los sistemas tradicionales de actualización (cursos, congresos, etc.) son importantes, pero no suficientes [5]. Ante esta situación, nuestros hallazgos muestran que posiblemente existen vacíos en la capacitación que reciben los estudiantes de ciencias de la salud por parte de la universidad.

En el estudio se encontró que el 60\% de los estudiantes consideraba que la preparación en estrategias de búsqueda de información era regular o deficiente, situación similar en los demás procesos estudiados (publicación, redacción, lectura crítica y metodología). Esta situación es más preocupante que la hallada por Molina et al [23] en estudiantes de medicina de la misma ciudad.

Con los resultados del estudio no se pueden atribuir las deficiencias en la búsqueda de información científica a la formación universitaria porque sólo se evaluaron los conocimientos, percepciones y prácticas de los estudiantes, y no otros componentes propios del currículo de la escuela o facultad.

Los conocimientos, prácticas y habilidades sobre la búsqueda de información en Internet en este grupo de estudiantes son regulares o deficientes, al igual que la percepción sobre la capacitación recibida y sobre su nivel de competencia en investigación. Por ello es necesario entrenar a los estudiantes en el proceso de investigación mediante programas de capacitación extracurricular y promover el estudio de estrategias pedagógicas para mejorar la capacitación universitaria en investigación.

\section{Bibliografía}

1. Gómez-Hernández JA. Alfabetización informacional: cuestiones básicas. URL: http://www.thinkepi.net/ alfabetizacion-informacional-cuestiones-basicas. [18.03.2009].

2. Sánchez-Tarragó N, Alfonso-Sánchez IR. Las competencias informacionales en las ciencias biomédicas: una aproximación a partir de la literatura publicada. ACIMED 2007. URL: http://bvs.sld.cu/revistas/aci/vol15_02_07/aci02207.htm. [18.05.2009].

3. Bravo R. La gestión del conocimiento en medicina: a la búsqueda de la información perdida. Anales del Sistema Sanitario de Navarra 2002. URL: http://www.cfnavarra.es/ salud/anales/textos/index25_3.html. [10.05.2009].

4. Cañedo-Andalia R. Hinari-PubMed: ¡Más de seis millones de artículos libres a texto completo en el área de la salud! ACIMED 2009. URL: http://bvs.sld.cu/revistas/aci/ vol19_4_09/aci02409.htm. [10.05.2009].
5. Rada-Gabriel G. Letelier ML. ¿Podemos mantenernos actualizados en medicina en el siglo XxI? Rev Med Chile 2009; 137: 701-8.

6. Castrillón-Estrada JA. García-Domínguez JC, Anaya-Taboada M, Rodríguez-Berdugo D, De la Rosa-Barranco D, CaballeroUribe CV. Bases de datos, motores de búsqueda e índices temáticos: Herramientas fundamentales para el ejercicio médico. Salud Uninorte Barranquilla (Col) 2008; 24: 96-119.

7. Gálvez MM. Publicaciones biomédicas: realidad de Chile y Latinoamérica. Rev Chil Radiol 2006; 12: 113-117.

8. Organización Panamericana de la Salud OPS/OMS La información científica en salud: acceso y utilización, capítulo 4. Las políticas públicas y los sistemas y servicios de salud. Salud en las Américas Regional 2007; I: 385-93.

9. Base de datos LILACS. Estadísticas de contribución 2008 y 2009. URL: http://bvsmodelo.bvsalud.org/site/lilacs/E/ Elilbvs3.htm. [20.06.2009].

10. Rodríguez CR. Motores de búsqueda sobre salud en Internet. ACIMED 2003. URL: http://scielo.sld.cu/scielo.php?script= sci_arttext\&pid=S1024-94352003000500002. [10.04.2009].

11. Núñez GM. Criterios para la evaluación de la calidad de las fuentes de información sobre salud en Internet. ACIMED 2002. URL: http://bvs.sld.cu/revistas/aci/vol10_5_02/ aci05502.htm. [10.04.2009].

12. Ávila de Tomás JF, Portillo-Boyero BE, Pajares-Izquierdo JM. Calidad en la información biomédica existente en Internet. Aten Primaria (Madrid) 2001; 28: 674-79.

13. Carnicero J. Luces y sombras de la información de salud en Internet. Sociedad Española de Informática de la Salud (SEIS) 2002. URL: http://www.seis.es/jsp/base.jsp?contenido=/jsp/ publicaciones/informes.jsp\&id=5.2. [22.06.2009].

14. Valenzuela JI, Camacho JC, Argüello A, Cendales JG, Fajardo R. Percepciones de los trabajadores del sector salud frente a Internet y las tecnologías móviles en Colombia. Rev Panam Salud Pública 2009; 25: 367-74.

15. Molina GAM, Dulzaides IME, Mora RO. Nivel de conocimientos sobre búsqueda de información en profesionales de la salud. MEDISUR 2007. URL: http://www.medisur.sld.cu/index. $\mathrm{php} /$ medisur/article/viewArticle/310. [10.05.2009].

16. Carranza-Lira S, Varela A. Aptitud para la lectura crítica de estudios de investigación clínica en médicos especialistas en adiestramiento. Ginecol Obstet Mex 2007; 75: 678-81.

17. Carranza-Lira S, Arce-Herrera RM, Gonzales-Gonzales P. Evaluación de una estrategia educativa para mejorar la aptitud para la lectura crítica de textos de investigación clínica en residentes de ginecoobstetricia de segundo año. Ginecol Obstet Mex 2007; 75: 661-6.

18. Valencia-Sánchez JS, Leyva-Gonzales FA, Jáuregui-Aguilar R. Degollado-Bardales L, Gómez-Arteaga GM. Competencia para la lectura crítica de informes de investigación clínica en residentes de cardiología. Rev Mex Cardiol 2009; 20: 35-41.

19. Ospina EG, Reveiz-Herault L, Cardona AF. Uso de bases de datos bibliográficas por investigadores biomédicos latinoamericanos hispanoparlantes: estudio transversal. Rev Panam Salud Pública 2005; 17: 230-6.

20. Cañedo AR, Mursuli HM. Determinación del grado de conocimiento sobre fuentes de información que poseen los especialistas en una entidad biomédica con labor docente e investigativa. ACIMED 2002. URL: http://bvs.sld.cu/ revistas/aci/vol10_5_02/aci01502.htm. [10.04.2009].

21. González RM, Santana-Arroyo S. Comportamiento de los estudiantes de medicina en la búsqueda de información en Internet. ACIMED 2008. URL: http://scielo.sld.cu/pdf/aci/ v17n4/aci09408.pdf. [10.04.2009].

22. Horna P, Curioso W, Guillén C, Torres C, Kawano J. Conocimientos, habilidades y características del acceso a Internet en estudiantes de medicina de una universidad peruana. Anales de la Facultad de Medicina 2002; 63: 32-9.

23. Molina OJ, Huamani C, Mayta-Tristán P. Apreciación estudiantil sobre la capacitación universitaria en investigación: estudio preliminar. Revista Peruana de Medicina Experimental y Salud Pública 2008; 25: 325-9. 
24. Huamani C, Chávez-Solís P, Mayta-Tristán P. Aporte estudiantil en la publicación de artículos científicos en revistas médicas indizadas en Scielo-Perú, 1997-2005. Anales de la Facultad de Medicina 2008; 69: 42-5.
25. Galán E, Manrique N, Villavicencio E, Yllatopa E, Peralta M, De la Cruz W. Producción científica de los investigadores del pregrado de medicina humana del Perú, 1993-2003. CIMEL 2005; 10: 41-8. 